\title{
Ignacio Chávez Rivera. Un paradigma en la medicina
}

\author{
Ignacio Chávez Rivera. A paradigm in Medicine
}

\section{Sergio Trevethan-Cravioto*}

Subdirección de la Coordinación de Enseñanza, Instituto Nacional de Cardiología Ignacio Chávez, Ciudad de México, México

\begin{abstract}
Resumen
Este documento sintetiza la vida de Ignacio Chávez Rivera, uno de los seis directores más valiosos que ha tenido el Instituto Nacional de Cardiología Ignacio Chávez Sánchez, su fundador y padre del ahora aludido. Su paso por la vida dejó un claro ejemplo a emular tanto en el ámbito científico, académico y docente como en los aspectos humano, familiar y social que lo convirtieron en el alumno más destacado del maestro Chávez. Su paso por la vida en la Academia Nacional de Medicina, la Universidad Nacional Autónoma de México, la Sociedad Interamericana de Cardiología, la Sociedad Mexicana de Cardiología y el propio Instituto ha dejado huella imperecedera en estas instituciones. El instituto Nacional de Cardiología se inclina reverente ante la figura de Ignacio Chávez Rivera.
\end{abstract}

Palabras clave: Semblanza. Paradigma. Ignacio Chávez Rivera. México.

\begin{abstract}
This article summarizes the life of Ignacio Chávez Rivera, one of the six most valuable directors that the National Institute of Cardiology has had "Ignacio Chávez Sánchez," founder of the same and father of the aforementioned. His time in life left a clear example to emulate both in the scientific, academic and teaching as well as in the human, family, social and friendly, which make him in the most outstanding student of Master Chávez. His time in life at the National Academy of Medicine, the National Autonomous University of Mexico, the Interamerican Society of Cardiology, the Mexican Society of Cardiology and the Institute itself, has left an indelible mark on these Institutions. The National Institute of Cardiology bows reverently to the figure of Ignacio Chávez Rivera.
\end{abstract}

Key words: Semblance. Paradigm. Ignacio Chávez Rivera. Mexico.

\section{Correspondencia:}

*Sergio Trevethan-Cravioto

E-mail: trevethan@ cardiologia.org.mx
Disponible en internet: 13-09-2019 Arch Cardiol Mex. 2020;90(1):77-80

www.archivoscardiologia.com 2604-7063/@ 2019 Instituto Nacional de Cardiología Ignacio Chávez. Publicado por Permanyer. Este es un artículo open access bajo la licencia CC BY-NC-ND (http://creativecommons.org/licenses/by-nc-nd/4.0/). 
Todos los que tuvimos el privilegio de conocer al mexicano ilustre (Fig. 1) que, entre otras tantas cosas, nos dejó como herencia el Instituto Nacional de Cardiología, que hoy orgullosamente lleva su nombre, consideramos un privilegio laborar en él y desde luego nos sentimos herederos de esta gloriosa tradición que lleva implícita la orden de continuarla, situación que ha ocurrido a través de los años desde la fundación del instituto en 1944 hasta la fecha. Sin embargo, el magno paradigma del cumplimiento de esta orden ocurrió de manera inusual en la persona de Ignacio Chávez Rivera, el propio hijo del maestro y uno de los seis directores que ha tenido el instituto.

En otras ocasiones, en las que por alguna razón he tenido que hacer uso de la palabra para describir la trayectoria y la personalidad de alguna celebridad, he señalado siempre que mi relación con ella se encontraba desprovista de cualquier afecto filial y que consecuentemente hablaba sólo del valor de la persona. Éste no es el caso cuando me refiero al Dr. Ignacio Chávez Rivera; a este hombre me une un sentimiento de afecto filial profundo e indestructible, basado en el reconocimiento de que le debo casi todo cuanto soy, que no es mucho, pero que para mí lo es todo y que deriva no del consejo, sino del ejemplo que me dio (sin que él se haya percatado y sin que jamás fuera su intento) con su trayectoria de vida, a través de una larga convivencia transcurrida a lo largo de 43 años, tiempo durante el cual he tratado de seguir su callada hidalguía.

Como dejé escrito en alguna ocasión ${ }^{1}$, es sin duda alguna Ignacio Chávez Rivera el mejor de todos los discípulos que haya tenido Ignacio Chávez Sánchez, (que fueron muchos y muy valiosos).

Distinguidas personalidades, como las de los doctores Jesús Kumate, Guillermo Soberón, Bernardo Fishleder, Daniel Cosío Villegas, Pierre Duchosal, Antonio Bayes de Luna, José Narro, Juan Ramón de la Fuente, Adolfo Martínez Palomo, y muchos otros más, han hablado y escrito acerca de la grandeza de la obra de Chávez hijo y la modestia con la que fue manejada.

En su carrera médica, después del título de médico cirujano obtenido en la Universidad Nacional Autónoma de México en 1952, se desarrolló como internista en el Instituto Nacional de Ciencias Médicas y Nutrición Salvador Zubirán del cual fue residente y se preparó en cardiología como residente en el Instituto Nacional de Cardiología entre los años 1954 y 1956; con posterioridad fue Research Fellow en la Universidad de Harvard en el Massachussets General Hospital de Boston en 1957 y de nueva cuenta Research Fellow en el Peter Bent Brigham Hospital de 1958 a 1959.

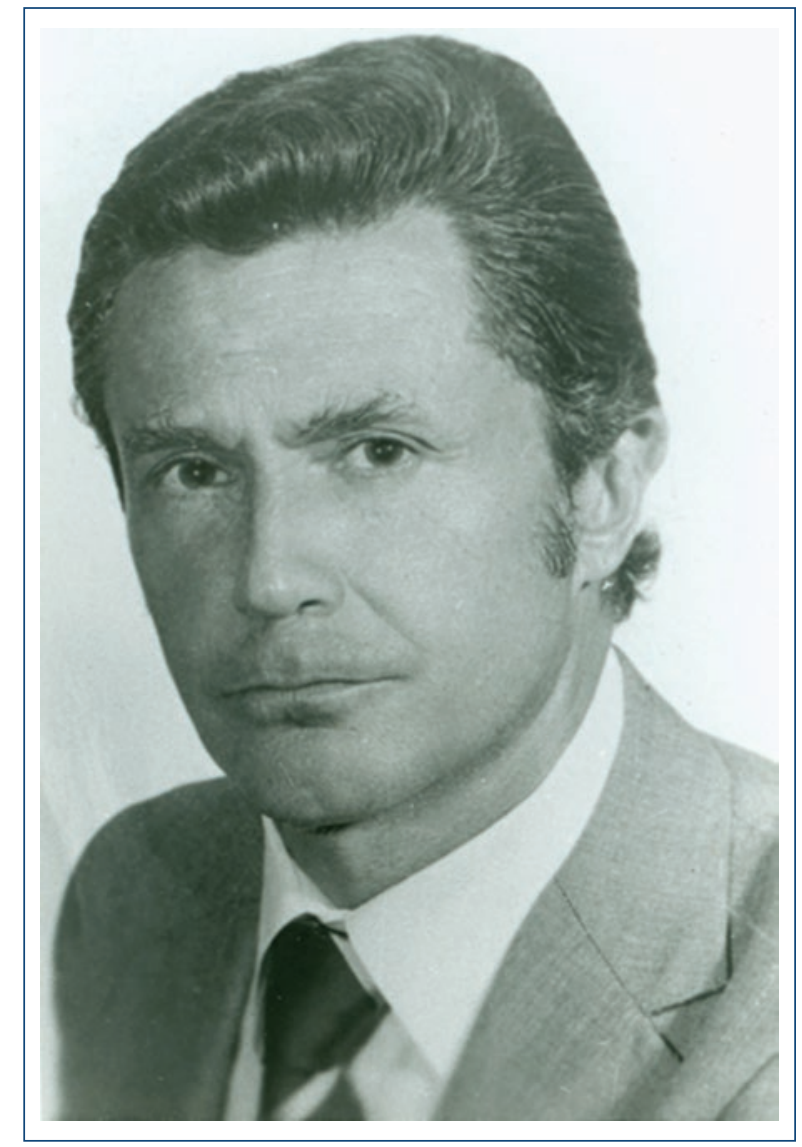

Figura 1. Dr. Ignacio Chávez Rivera.

Desde temprana edad, la producción científica de Ignacio Chávez Rivera empezó a fructificar en numerosas publicaciones y en la creación de grandes obras cardiológicas que han quedado para la posteridad: Coma, síncope y shock, libro de 400 páginas editado en 1969 con reediciones en 1974 y 1976², Cardioneumología fisiopatológica y clínica de $1973^{3}$ obra "titánica y enciclopédica" en dos volúmenes con 2,000 páginas y considerada en todo el mundo como obra de consulta obligatoria y fundamental de la cardiología, Cardiopatía isquémica por ateroesclerosis coronaria 4 , libro de 518 páginas editado en 1979 y reeditado en 1982, Hipertensión arterial esencial ${ }^{5}$ de 1984 y reimpreso en 1985 , un libro de 300 páginas, Cardiopatía coronaria e isquemia miocárdica ${ }^{6}$ de 1989 y Cardiología $^{7}$, dos volúmenes de 1,600 páginas editado en 1993.

En la obra de Ignacio Chávez Rivera destaca el academicismo de manera fundamental. En su ascendente carrera fue distinguido como Subjefe de médicos residentes en el Instituto Nacional de Cardiología en 1955 y Jefe de médicos residentes en el 1956. Desde Médico Adjunto, Jefe de Servicio Clínico, Jefe de la División de Enseñanza y hasta Director General del Instituto en 
dos periodos renovados de 1989 a 1999.Tuvo más de 300 participaciones como conferencista invitado en la Ciudad de México, 100 más en toda la República Mexicana y en 10 países de América y Europa. Numerosas ponencias en Congresos y Jornadas Médicas, 30 artículos de investigación clínica, 66 de revisión de concepto o difusión y otros 200 diversos. Es coautor en seis libros. Fue Secretario Tesorero de la Sociedad Interamericana de Cardiología, Presidente de la Sociedad Mexicana de Cardiología, que más adelante lo designó como miembro honoris causa. Además, su destreza y afición por el dibujo lo llevó a colaborar en el excelente libro de ilustraciones médicas de Frank $\mathrm{H}$. Netter ${ }^{8}$.

Fue presidente de la segunda junta de gobierno del Consejo Mexicano de Cardiología; miembro asociado por invitación u honorario en 15 sociedades médicas en diferentes países; miembro de la Academia Nacional de Medicina desde 1970; secretario de ésta en 1971, y presidente en 1985.

En el seno de nuestra Universidad Nacional Autónoma de México, el Dr. Chávez tuvo una destacada labor, desde profesor de la asignatura en cardiología, profesor por oposición y titular de posgrado hasta miembro de la junta de gobierno de dicha casa de estudios de 1985 a 1997.

De vida modesta a pesar del entorno que lo rodeaba y habiendo nacido en el seno de una familia ilustre y muy conocida ya en el ámbito intelectual y social del México de la primera mitad del siglo pasado, y a diferencia de lo que ocurre con frecuencia, a Ignacio Chávez Rivera no le afectaba esto de modo desfavorable; por el contrario, le confirmaba más su idea de mantenerse callado, lejos de la presunción y el elogio y sobre todo del autoelogio que tanto se mira en la actualidad.

En Ignacio Chávez Rivera se viven, como en ningún otro de los discípulos del maestro Ignacio Chávez Sánchez, la honestidad, la modestia, la decencia y la lealtad, enlazando siempre (como señalaba el mismo maestro), la acción y el pensamiento.

En Ignacio Chávez Rivera se verifica también de manera cabal el dicho popular que reza: "Que el mejor predicador es Fray ejemplo", prueba de lo cual son su actuar y su comportamiento. Siendo él director del Instituto, su modesto automóvil nunca estuvo estacionado en el lugar reservado para la dirección, sino en el estacionamiento general. $\mathrm{O}$ bien, habiendo comedor particular para los miembros de la dirección, siempre prefería comer en el comedor general acompañado de otros médicos, los adjuntos, los residentes, las enfermeras y aun del resto del personal administrativo o de la intendencia.

En algunas ocasiones su natural modestia fue confundida o mal entendida por algunos como falta de carácter o timidez, sobre todo por los que tuvieron menor talla intelectual que la de él.

No existe mayor carga que pueda llevar un hombre sobre sus espaldas toda una vida que la de ser hijo de uno de los hombres más ilustres que ha tenido nuestro país y, al mismo tiempo, ejercer la misma profesión del padre. El Dr. Chávez cumple callada, tranquila y serenamente su misión en la vida, soportando sobre sus hombros esta enorme cruz, con un gran orgullo interno sí, pero siempre sin abusar de privilegios, canonjías o prebendas que le habrían dispensado ser hijo de quien fue, sino con trabajo cotidiano, esmerado, dedicado y plagado del humanismo que le fue legado.

Que no se entienda mal ni por asomo algún descrédito que mis palabras pudieran sugerir para la eminencia del maestro Chávez Sánchez, quien además de todas sus virtudes y cualidades, como diría Bertrand Russell, el Maestro Chávez tenía una cualidad llamada brillo; sin embargo, es preciso señalar que los tiempos que les tocó vivir a Chávez padre y a Chávez hijo son totalmente diferentes, al igual que las oportunidades de transformación del medio. El México de los años de Chávez padre apenas florecía en la Posrevolución y con él una pléyade de intelectuales probos, bien intencionados, que formaron el grupo de sus primeros amigos en Morelia, haciendo honor al viejo dicho de que "Dios los creó y ellos se juntan" y otros posteriores como fueron los forjadores del Colegio Nacional.

En los logros del Instituto Nacional de Cardiología de la época de Chavez padre (lo señalaba él mismo) intervino cierta autonomía de gobierno que le fue permitida, pese a ser dependencia gubernamental de la Secretaría de Salud del gobierno federal de la República Mexicana, con la cual la Dirección de Chávez hijo ya no contó y, por el contrario, el mundo de Chávez hijo está plagado de sobrepoblación, pobreza, corrupción, menester social al por mayor y criminalidad desmedida, todo ello aunado a una excesiva burocratización de la instituciones gubernamentales. En el México actual, las personas no quieren ser, sólo quieren tener a costa de lo que sea.

Los valores de antaño han sido reemplazados. La mortalidad infantil ha disminuido de manera notable y, de la misma manera, la esperanza de vida para los viejos se ha prolongado de forma inusitada; cada vez nacen más y mueren menos, mientras que el tamaño del pan parece ser el mismo y muy pocos aportan para 
que éste crezca. Es probable que la sociedad científica de un futuro no lejano y las instituciones religiosas recapaciten seriamente sobre esto y sobre el derecho a tener tantos hijos como la naturaleza y la inconciencia decidan.

Chávez Rivera dejó un caudal enorme de producción académica a las nuevas generaciones, que rebasó con mucho la obra académica escrita por su padre y que, para los que escribimos aun en muy pequeña escala, conocemos bien el esfuerzo, la dedicación y el trabajo que esto representa.

Entre sus múltiples cualidades figuran de manera notable la buena educación y la decencia y, en forma muy destacada, su habilidad para conocer el trasfondo de las diferentes personalidades, capacidad que le permitió en sus gestiones como director de nuestra casa y como presidente de la Academia de Medicina o en la Junta de Gobierno de nuestra universidad, delegar las diferentes responsabilidades en los hombres idóneos para cada mando, sin nepotismos, sin mimetismos, sin favoritismos, sin falsedades $y$, sobre todo, sin retórica.

Su concepto de la familia es también por demás envidiable, así como su fidelidad, lealtad y ejemplo transmitidos a sus hijos. Las decisiones que tomó Chávez Rivera nunca fueron atropelladas, nunca de manera arbitraria y jamás sin reflexión previa. En la vida de Ignacio Chávez Rivera destaca de manera promitente su gran compañera de toda la vida, Ofelia de la Lama de Chávez, mujer brillante, de intelecto agudo, cultura amplia y seleccionada, pero sobre todo de actitud positiva en la vida, especialmente en las adversidades. Ambas personalidades, la de Chávez y la de Ofelia, se complementaron y cristalizaron en una hermosa familia de universitarios, cultos y destacados.

El paso por la vida de Ignacio Chávez Rivera fue intachable y su valor moral imperecedero; los que tuvimos la fortuna de estar cerca de él enriquecimos sin duda alguna nuestro espíritu, nuestra moral y nuestra cultura.

Pero además, si hay algo digno de admiración y respeto en la vida de Chávez, es su absoluta congruencia, de acuerdo con los preceptos dictados por su padre de fusionar la acción y el pensamiento. El maestro
Ignacio Chávez Rivera cumplió con creces la promesa hecha a su padre de labrarse él solo un nombre propio en la medicina y en la sociedad.

Maestro Ignacio Chávez Rivera, estimado e inmerecido amigo, le saludo.

\section{Sergio Trevethan Cravioto}

Subdirector de la Coordinación de Enseñanza, Instituto Nacional de Cardiología Ignacio Chávez.

\section{Financiamiento}

No se recibió ningún financiamiento para la redacción de este artículo.

\section{Conflicto de intereses}

El autor declara no tener ningún conflicto de intereses.

\section{Responsabilidades éticas}

Protección de personas y animales. Los autores declaran que para esta investigación no se han realizado experimentos en seres humanos ni en animales.

Confidencialidad de los datos. Los autores declaran que en este artículo no aparecen datos de pacientes.

Derecho a la privacidad y consentimiento informado. Los autores declaran que en este artículo no aparecen datos de pacientes.

\section{Bibliografía}

1. Trevethan-Cravioto S. Ignacio Chávez y la Escuela Cardiológica Mexicana e Internacional: el pensamiento médico contemporáneo. En Uribe-Elías R (coord.). Aguascalientes: Universidad Autónoma de Aguascalientes, 2007:81-98.

2. Chávez- Rivera I. Coma, síncope y shock. Ciudad de México: Francisco Méndez Otero, editor y distribuidor, sucursal Mérida, 1969.

3. Chávez-Rivera I. Cardioneumología fisiopatológica y clínica. Ciudad de México: Dirección General de Publicaciones, Universidad Nacional Autónoma de México. Ciudad Universitaria, 1973.

4. Chávez Rivera I. Cardiopatía isquémica por aterosclerosis coronaria. Ciudad de México: Salvat Mexicana de Ediciones, 1978.

5. Chávez-Rivera I. Hipertensión arterial esencial. Ciudad de México: Ediciones Crossiere, 1985.

6. Chávez Rivera I. Cardiopatía coronaria e isquemia miocárdica. Ciudad de México: McGraw-Hill-Interamericana, 1989.

7. Chávez-Rivera I Cardiología. Ciudad de México: Lito Arte, 1993.

8. Netter F. The Ciba Collection of Medical Ilustrations. Vol. 5 Heart. New Jersey: 1978. 